Proceedings

\title{
Deep Neural Network Recognition of Shallow Water Corals in the Gulf of Eilat (Aqaba) ${ }^{\dagger}$
}

\author{
Alina Raphael 1,*, Zvy Dubinsky ${ }^{1}$, David Iluz ${ }^{1,3}$, Jennifer I. C. Benichou ${ }^{1}$ and Nathan S. Netanyahu ${ }^{2}$ \\ 1 The Mina and Everard Goodman Faculty of Life Sciences, Bar-Ilan University, \\ Ramat-Gan 5290002, Israel; dubinz@mail.biu.ac.il (Z.D.); iluzda@gmail.com (D.I.); jeniiii.bee@gmail.com \\ (J.I.C.B.) \\ 2 Department of Computer Science, Bar-Ilan University, Ramat-Gan 5290002, Israel; nathan@cs.biu.ac.il \\ 3 Department of Environmental Sciences and Agriculture, Beit Berl College, Beit Berl 4490500, Israel \\ * Correspondence: alina.raphael@live.biu.ac.il (A.R.); creativeacs@gmail.com (X.X.); \\ Tel.: +972-3-972-3-5318283; Fax: +972-3-7384058 \\ + Presented at the 1st International Electronic Conference on Biological Diversity, Ecology and Evolution, \\ 15-31 March 2021; Available online: https://bdee2021.sciforum.net/.
}

Citation: Raphael, A.; Dubinsky, Z; Iluz, D.; Benichou, J.I.C.;

Netanyahu, N.S. Deep Neural Network Recognition of Shallow Water Corals in the Gulf of Eilat (Aqaba). Proceedings 2021, 68, x. https:// doi.org/10.3390/xxxxx

Published: date

Publisher's Note: MDPI stays neutral with regard to jurisdictional claims in published maps and institutional affiliations.

Copyright: (c) 2021 by the authors. Submitted for possible open access publication under the terms and conditions of the Creative Commons Attribution (CC BY) license (http://creativecommons.org/licenses/by/4.0/).

\begin{abstract}
We describe the application of the deep learning computerized methodology to the recognition of corals in a shallow reef in the Gulf of Eilat, Red Sea. This project aimed at applying deep neural network analysis, based on thousands of underwater images, to the automatic recognition of some common species among the 100 species reported to be found in the Eilat coral reefs. This is a challenging task, since even in the same colony, corals exhibit significant within-species morphological variability, i.e., age, depth, current, light, geographic location, and inter-specific competition. Since deep learning procedures are based on photographic images, the task is further challenged by image quality, distance from the object, angle of view, and light conditions. We produced a large dataset of over 5000 coral images that were classified into eleven species used in the present deep learning machine classification. We demonstrate the efficiency and reliability of the method, as compared to painstaking manual classification. We demonstrated that this method is readily adaptable to include additional species, providing an excellent tool for the benefit of future studies done in the region, allowing real time monitoring the detrimental effects of global climate change and anthropogenic impacts on the coral reefs of the Gulf of Eilat and elsewhere, and assessing the success of bioremediation efforts.
\end{abstract}

\section{Introduction}

One of the major challenges in the field of contemporary ecology is the documentation of ecosystem change over time. Among coastal marine biota, coral reefs are home to a unique hotspot of biodiversity. In the last decades, coral reefs are undergoing a severe decline worldwide [1-3] due to a combination of ocean acidification [4,5], and seawater warming [6,7], their adverse impacts intensified by anthropogenic eutrophication and pollution [8]. These bring about both the decline in live reef cover and a decrease in coral species diversity [1]. Hence it is of paramount importance to monitor and document the rates of reef decline and identify the relative importance of stressors in each reef. An additional benefit of automated analysis of reef images is its potential as a tool to evaluate the long-term success of bioremediation projects of damaged coral reefs [9] and reef protection measures.

The use of artificial intelligence (AI) to solve the time-consuming, tedious manual classification of coral species and determination of their abundance in real-time, is a Herculean task by itself due to the immense numbers of necessary images and their examination. The automated Deep learning (DL), a branch of AI, has the potential of solving this problem efficiently, far exceeds in reliability and accuracy human reef documentation and 
monitoring. Like the human brain, the more data the computer learns under the DL program, the better it becomes at distinguishing among classes of coral species in the present application.

The highly diverse of the Gulf of Eilat (Aqaba) coral reefs are of special scientific interest as one of the most Northern reefs, as well since they are the main basis of the economy of surrounding communities in both Israel and Jordan. They are highly diverse [10], but have suffered from a sequence of disasters, including a rare low tide in 1970 [11], the recovery from which was impeded by repeated oil pollution following the closure of the Suez Canal between 1967 and 1975 [12]. The subsequent recovery of the gulf's reefs was slowed down again by the episode of the cooling of the gulf's waters due to the eruption of Mount Pinatubo in 1991. That event caused erosion of the gulf's thermocline and led to deep mixing of its waters, enriching surface waters with nutrients. The resulting proliferation and subsequent decomposition of seaweeds smothered some $25 \%$ of the juvenile corals [13]. The anthropogenic eutrophication of the gulf due to the increase in fish farming until the farm closing [14], also reduced the transparency of its waters by increasing the concentration of phytoplankton [15]. These events, as well as the forthcoming RedDead Canal, call for frequent, detailed monitoring of any changes in the situation of the coral reefs of the Gulf, as are evident in the reduction of live coral cover and species biodiversity.

Since its early development, DL has been used in human facial discrimination [16], handwriting recognition [17], and forensic applications, such as fingerprint identification [18] and voice analysis [19].

DL has already been applied to coral reef studies, in which it was used to discriminate among benthos types: sand, urchins, and three types of branched corals: brain coral, massive favids, and dead coral [20], as well as to distinguish between healthy and bleached corals (see, e.g., [21,22]. Shihavuddin et al. [20] demonstrated the capability of DL to identify five coral genera from large assemblages of underwater images. In a recent study identification among branched and brain corals, was reported [23].

In their research, Gómez Ríos et al. [23] also distinguished among favids, brain coral, and three branched coral types: I, II, and III (an urchin, dead corals, and pavements based on an image mosaic).

Among DL studies at species level discrimination the following datasets are noteworthy:

The first dataset is the Pacific Labelled Corals dataset. That contains 5090 images from four locations: (1) Mo'orea (French Polynesia), (2) Northern Line Islands, (3) Nanwan Bay (Taiwan), and (4) Heron Reef (Australia). Pacific Labelled Corals dataset contains: 251,988 annotations from these four locations done by a coral reef expert using a random point tool. In addition six experts cross-annotated 200 images from each location [21].

The second dataset is the Mo'orea Labeled Corals (MLC) dataset that includes five coral classes: Acropora, Pavona, Montipora, Pocillopora, and Porites, and four non-coral classes: crustose coralline algae, turf algae, macroalgae and sand are included in the Mo'orea Labeled Corals (MLC) dataset containing over 400,000 human expert annotations of 2055 Mo'orea island survey images (http://vision.ucsd.edu/datasetsAll).

For DL of a reef, there is no need to sample small fragments of corals for the subsequent tedious identification in the laboratory. DL enables classification to be done directly on a large amount of photographs, in minimum time.

The novelty of the present study is applying DL, based on imaging, and its fully automated methodology to recognize eleven common coral types from among the species reported in the Gulf of Eilat [11]. We applied DL to over 5000 underwater images taken specifically by us from a shallow reef in the Gulf of Eilat, with the aim of documenting the distribution of the test types in the sample reef.

We demonstrated the power of DL, using shallow coral reefs in the Gulf of Eilat for comparison with its efficiency in other reef sites. That site allowed us to compare our DL data with the detailed manual coral surveys previously conducted on these reefs. 


\section{Corals and Reefs}

The nature and global importance of corals and the rapid destructive impact of Global Climate Change call for extensive and fast indexing and monitoring. Coral reefs cover less than $1 \%$ of the total area of the oceans and seas, yet they are the main repository of oceanic biodiversity (25\% of all marine species) [24]. Extant hermatypic (reef-building) coral species are estimated at 3235 [24], of which 100 were recorded in Eilat, Gulf of Aqaba, Northern Red Sea [10]. Hexacorals, based on six fold symmetry, or scleractinian corals are the most important hermatypic organisms [25].

The decline of reefs leads to the collapse of their entire complex ecosystem depending on the calcium carbonate skeletons of the corals intricate reef structures, for food and shelter. Hermatypic corals are home to symbiotic algae living within their cells in specialized organelles, the symbiosomes. Called zooxanthellae by their first reporter, Brandt [26], these greenish microalgae limited to sunlit shallow waters $(\sim 0-120 \mathrm{~m})$, provide the corals with energy through their photosynthesis [27,28], which also stimulates calcification [29].

In most corals, the tentacles are retracted by day and spread out at night [30] to catch plankton and other small organisms, while avoiding diurnal coral-feeding predators. This behaviour also optimizes the supply of oxygen for nocturnal respiration.

Unlike in shallow water, corals satisfy their energy needs in the deep water and dim light by zooplankton consumption, as an energy supplement to the algal light-limited photosynthetic products see review by Dubinsky and Iluz [28].

The photosynthetic activity of the zooxanthellae, raises the internal $\mathrm{pH}$ of the coral facilitating the skeletal calcification by "light enhanced calcification" [31,32], a paradigm recently challenged by Cohen et al. [33]. Conversely, ocean acidification makes coral calcification more difficult.

\section{The Future of Coral Reefs}

Coral reefs are exposed to many dangers because of global climate-change effects [34,35], blast and cyanide fishing [36], coral collection the marine coral aquarium trade [37], sunscreen use [38], and light pollution interference with lunar cycle reproduction timing [39]. SCUBA diving pressure [40]. Anthropogenic eutrophication, acts synergistically with all the above listed detrimental factors, stimulating fast seaweed growth, that easily outcompete the slowly growing corals. The ensuing algal blooms, smother the coral colonies and prevent the settlement of juveniles [41]. Kaneohe Bay, a coral reef ecosystem at Oahu, Hawaii, illustrates the sensitivity of coral reefs to nutrient enrichment resulting from treated sewage disposal, leading to the reversible proliferation of seaweeds [42]. Fish cage farming released nutrients that affected the coral reefs in Eilat by causing deterioration in water quality due to eutrophication and by promoting seaweed growth and phytoplankton proliferation reducing the Gulf's water transparency, thus reducing light necessary for symbiont photosynthesis, interfering with reproduction, increasing bio-erosion and epizootic infestation [14].

Coral species differ in their tolerance to climate change and coral bleaching [43]. Corals experience bleaching as water temperature increases and causes loss of the zooxanthellae, and subsequently of live coral tissue, resulting in wide spread coral mortality followed by reef destruction. Unless the algal population recovers within weeks, the bleaching results in widespread reef mortality [44]. The ongoing increase in atmospheric carbon dioxide since the industrial revolution leads to ocean acidification or lowering of ocean $\mathrm{pH}$, and affects corals negatively by shifting the balance from skeletal aragonite deposition toward its dissolution [4]. In addition, light pollution by artificial light, even at the weakest intensities [45,46], can cause the disruption of coral reproduction that is controlled by lunar periodicity [47,48]. The planned Red Sea-Dead Sea Conveyance [49] will cause a change in the regime of the gulf currents [50]. Such a change could reduce the supply of larvae of corals and other reef organisms, and have a far-reaching deleterious impact on reef systems. 
The real-time characteristics of DL tools are crucial for the rapid detection of reef damage allowing implementation of bioremediation measures. The DL characteristics are valuable tools assuring the health and long-term survival of the coral reefs in the Gulf of Eilat and worldwide.

\section{Deep Learning}

The efficiency of the methodology of DL, for the classification of coral species consists of algorithms that reveal and extract common-patterns and features from large image datasets. Two popular algorithms applied to coral reef data are the convolutional neural network (CNN) [21] and deep belief net (DBN) [21]. A generic structure of CNN is a multilayer, feed-forward, supervised neural network that recognizes objects from spatial-based images with little or no pre-processing. It consists: (1) feature extraction (convolution layer); (2) distortion invariance (sub-sampling layer); and (3) classification (output layer). DBN, consists of probabilistic models composed of multiple layers of random variables [51].

Any coral-reef classification consists of five main steps:

1. Taking sufficient high quality underwater images

2. Detecting the chosen coral and cropping its image

3. The cropped images are scaled to $200 \times 200$ pixels

4. Images are pre-processed to compensate for different imperfections (blurring, colour change, sunlight wave patterns, sky colour, nekton scattering effects etc.

5. After pre-processing, each one of the coral species is labelled.

In the case of an automatic model Steps 3 and 4 are not required.

Traditional machine learning methods need extensive domain expertise, human intervention, and are only capable of what they were originally designed for.

Additional works on growth modelling and quantification of morphological variation in coral types (see, e.g., Kruszyński et al. [52]; Chindapol et al. [53]) Kruszyński et al. studies focused on analysis of three-dimensional (3D) images of corals scanned by X-ray Tomography.

Chindapol et al. modelled the effects of flow on colony growth and shape, using analyzed advection-diffusion equations.

The increased interest in DL has also been recently reflected in the analysis of previously published coral datasets. Specifically, recent work [22] has demonstrated the efficiency of neural networks and DL in distinguishing among various marine benthos components such as bare ground, seagrass meadows, algal cover, sponges, and identified some coral species. Additional recent work has shown the capability of neural networks and DL to distinguish among coral species and live corals from bleached colonies (see, e.g., $[21,22])$.

CNN representations were coupled by Mahmood et al. [22] combined with manually obtained colony parameters. They used algorithms based on the information present in the image. These authors extracted from VGGnet CNN images with a 2-layer multilayer perceptron (MLP) classifier [trained with the Mo'orea Labeled Corals (MLC) dataset]. They achieved $77.9 \%$ accuracy.

Mahmood et al. [54], reviewed the power of DL for machine monitoring of coral reefs.

Mahmood et al. (2016) [55] reported a decrease trend in coral density and species numbers of the of the Abrolhos Islands reefs. Their analysis was based on pre-trained CNN images from VGGnet. They proved the reliability of their classifier on unlabelled coral image mosaics.

Mahmood et al. (2018) [56] used CNN based features and ResFeats to annotate corals and demonstrated the temporal changes in their association. They applied generic features from VGGnet and ResNet to classify corals and non-corals. They analysed unlabeled coral mosaics of three Abrolhos Island sites generating maps for the aforementioned mosaics. 
Mahmood et al. (2020) [57] applied DL computerized characterization of annotation of

kelp species. They presented an automatic hierarchical classification method to classify kelps in images collected. That study summarises the considerable advantages of using deep residual networks (ResNets) over traditional, manual classifications of the same reefs. They showed that the sibling hierarchical training approach outperforms the traditional parallel multi-class classifications by a significant margin $(90.0 \%$ vs. $57.6 \%$ and $77.2 \%$ vs. 59.0\%) on Benthoz15 and Rottnest datasets. They used an application to study the changes in kelp cover over time for annually repeated AUV surveys.

Mahmood et al. (2020) [58] evaluated how well features extracted from deep neural networks, transfer to underwater image classification. They investigated the effectiveness of transfer learning of the ResFeats. They proposed new image features (called ResFeats) extracted from the different convolutional layers of a deep residual network pre-trained on ImageNet on MLC, Benthoz15, EILAT and RSMAS datasets.

Gómez-Ríos et al. [23] included more corals than previous studies by applying three CNNs: Inception v3 [59], ResNet [60], and DenseNet [61] (Supplementary Table S1).

Two datasets were analysed: EILAT and RSMAS, both of which comprised patches of coral images discriminating branched and massive colonies. EILAT contains eight classes (sand, urchin, branched type I, II, and III corals, brain coral, favid coral, and dead coral) and 1123 images. RSMAS contains 14 classes, including 9 classes of the following scleractinian coral species: Acropora cervicornis, Acropora palmata, Diploria strigosa, Montastraea cavernosa, Meandrina meandrites, Montipora spp.; Siderastrea sidereal, Colpophyllia natans (a boulder brain coral), and the colonial fire coral Millepora alcicornis (a species of hydrozoa with a calcareous skeleton). The other five classes included in the RAMAS dataset are not coral species: Diadema antillarum is a sea urchin, Gorgonians are a genus of soft corals in the family Gorgoniidae, Palythoas palythoa is a genus of anthozoans in the order Zoantharia, and sponge fungus and tunicates are marine invertebrates of the subphylum Tunicata. The RSMAS dataset includes 776 images.

CoralNet was conceived by Beijbom et al. [9,62]). In early 2019, Williams et al. [63] in a large study showed that the automated annotations for CoralNet Beta, produced benthic cover estimates comparable to controls gathered by human annotation.

CoralNet (https://coralnet.ucsd.edu/) uses deep neural networks for fully or semiautomated annotation of images. It also serves as a convenient, user-friendly collaboration platform.

HoeghGuldberg states that "CoralNet will allow the world's scientists to quickly assess the health of endangered coral reefs at scales never dreamed of before", in (https://blogs.nvidia.com/blog/2016/06/22/deep-learning-save-coral-reefs/).

BenthoBox image labelling system for ecologists allows storing images of the dataset.

The software uses learning algorithms to recognise 'tagged' seabed features such as sand, algae, sponges and corals.

\section{History of Coral Classification in the Gulf of Eilat}

Traditional methods have been used in Gulf of Eilat research studies for coral classification since the pioneering work by Loya and Slobodkin [10]. Some 100 coral species were listed in their study.

Whenever confronted with doubt concerning the species of a certain coral underlying a transect, a small piece was sampled and manually identified by a taxonomist [11], a tedious and destructive practice based on limited sample size.

These surveys were based on colour photographs taken by a camera with a flash attachment. Close-ups were taken by a Rolleiflex camera. A measuring tape was spread over the reef, and the divers recorded the projected length of all the organisms and substrates underneath the line transect to a resolution of $1 \mathrm{~cm}$. Photographs were taken at $1 \mathrm{~m}$ intervals along the transect. This study was based on permanent transects photographed over a period of 20 months that yielded about 3000 photographs of corals belonging to Loya's 
[11] list of approximately 100 species. However, the author noted that many cryptic species do not show up in the photographs.

Similar additional surveys were conducted following various disturbances that affected the coral reefs of the gulf: the 1970 low tide [64], the repeated oil spills [65], the Pinatubo eruption of 1991 [66], and the fish farming episode of 1995-2008 [14].

Diver-based methods for classifying corals are almost impossible underwater, and require time-consuming expertise. Furthermore, coral pigmentation and morphology are plastic changes in response to environmental forcing functions such as light and current, eliciting wide phenotypic variability [28,67]. Ever since the National Monitoring Program (http://www.iuieilat.ac.il/Research/NMPmeteodata.aspx) of the Eilat reefs was initiated (2003), annual surveys by divers have been conducted.

The images are taken at a fixed area at six reef sites, namely the North Beach, the Dekel Beach, the Eilat Ashkelon Pipeline Co. Ltd. (EAPC), the coral reserve, the Interuniversity Institute for Marine Sciences in Eilat (IUI) marine laboratory, and Taba. Each site has fixed camera brackets for five cameras, and each of these takes four images. In this way, 20 pictures are taken at each site, and 120 pictures are taken for quantitative analysis of the changes at the various sites. Monitoring is done once a year in early summer. Corals were identified as far as possible at the species level, and were also classified according to functional groups. The results are presented graphically following statistical processing. Due the disintegration of the rock to which the cameras were attached, some new sites had to be added [68].

Automated DL seeks to avoid these difficulties, profiting from the latest advances in computerized handling of large quantities of visual images [9]. Indeed, these novel developments have been increasingly applied to the survey and analysis of coral reefs in the studies listed in Supplementary Table S2. Since all previous surveys, as well as those of the current monitoring program of the Gulf of Eilat reefs, were based on the manual and visual analysis of large numbers of photographs, we present here a first example at using automated machine-based analysis for the red sea coral reef.

\section{Methods}

\subsection{Work Process}

The photos and underwater videos of transects were acquired at the coral reef reserve in the Gulf of Eilat $\left(29^{\circ} 30^{\prime} \mathrm{N}, 34^{\circ} 55^{\prime} \mathrm{E}\right)$.

The methods used in the current study are:

a. Natural sampling units by photographing the coral reef during daytime.

b. Line transects for estimating the cover percentage at the four test sites in the Gulf of Eilat.

c. Deep convolutional neural networks as an efficient classification for coral species using a supervised DL method called convolutional neural networks (CNNs).

d. The Cochran-Mantel-Haenszel test was performed to compare the presence and proportions of coral species abundance, as measured by different methods across multiple sites. Post hoc analysis was performed with pairwise Fisher test with false discovery rate (FDR), which is the expected proportion of type I errors.

Species coverage percentage was estimated using a one-way ANOVA, followed by Tukey post hoc analysis.

\subsection{Study Sites}

Field work at four sites (Figure 1) was conducted from June 2017 till June 2018 in Eilat Coral Beach Nature Reserve. This site has a well-developed reef near the shore, as well as massive stony corals throughout the entire depth gradient down to $50 \mathrm{~m}$. This is the most developed, complex, and diverse coral reef in the Gulf of Eilat. 


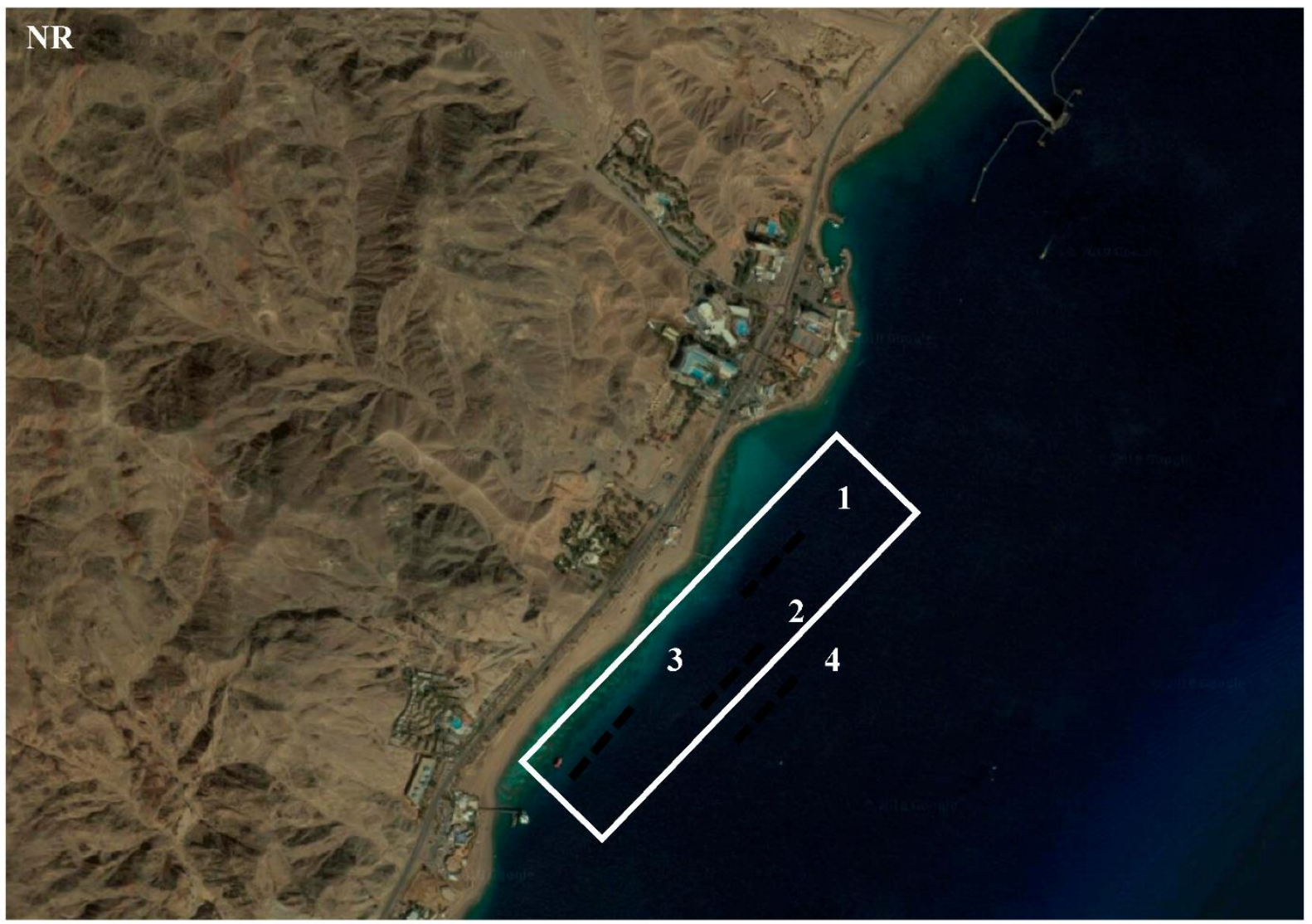

Figure 1. Location of study areas (white bars) at the nature reserve (NR). Figure 1 was generated from Google Maps version number: 10.26.2, URL: https://goo.gl/maps/arRzA1ZmbZvjwVJp7. Map data (02019 Mapa GISrael, ORIONME Imagery @2019 , CNES/Airbus, Landsat/Copernicus, Maxar Technologies, U.S. Geological Survey.

Two field data acquisition methods were used in this research:

- "Natural sampling units" by photographing the coral reefs.

- Line transects for estimating the cover percentage at the four sites in the Gulf of Eilat.

The study sites were chosen on the basis of their accessibility and central location within the Eilat Coral Reserve. Furthermore, they are highly diverse, offering the opportunity to choose the most common species. The chosen sites allow studying the variability over space (between sites), and finally, examining the possible effects of human-mediated disturbances by comparing quantity and cover percentage at the most disturbed site of the three with reference sites at different depths.

\subsubsection{Photography}

More than a thousand still coral images were taken, and hours of underwater videos were recorded. The first step began by the underwater photographing of 400 still images, each covering about $1 \mathrm{~m}^{2}$ of the reef area. Subsequently, squares of $200 \times 200$ pixels containing any of the 4 coral genera chosen for the initial stage of the study (Acropora, Favia, Stylophora, and Platygyra) were identified visually on the computer screen, labelled, and cut out of the original images.

\subsubsection{Equipment}

Photographing corals at the surveyed sites was done along line transects using an underwater Hero6 Black camera that offers video shooting at maximum resolution of $4 \mathrm{~K}$ at 60 frames per second, and also supports 1080p FHD 1080P video playback, or 2.7K at 
120 frames per second. The camera has video stabilization capabilities, as well as the ability to download images from the camera to a computer or smartphone through a $5 \mathrm{GHz}$ WiFi connection. It also has a GPS component, accelerometer, and gyroscope.

\subsubsection{Transects}

Each site and depth were marked by four line transects. At each site or depth, a $40 \mathrm{~m}$ line was laid parallel to the coastline from a randomly chosen point, and four sections were marked along the line at $10 \mathrm{~m}$ intervals, i.e., each section was $2.5 \mathrm{~m}$. The four transects corresponded with 5, 10, 15, and $20 \mathrm{~m}$ depth. Every line transect produced hundreds of still photos and thousands of video frames.

\subsubsection{Areal Coverage}

In the present study, the coverage percentage of the corals serves as an indicator of the coral reef's health. Throughout the study, the relative coverage of eleven common species was recorded.

The results are divided into two sections:

1. Coral species quantities and coverage percentage at each site (sites 1-4) by two methods (point estimated, Fiji ImageJ) (see Figure 2).

2. DL coral classification data.
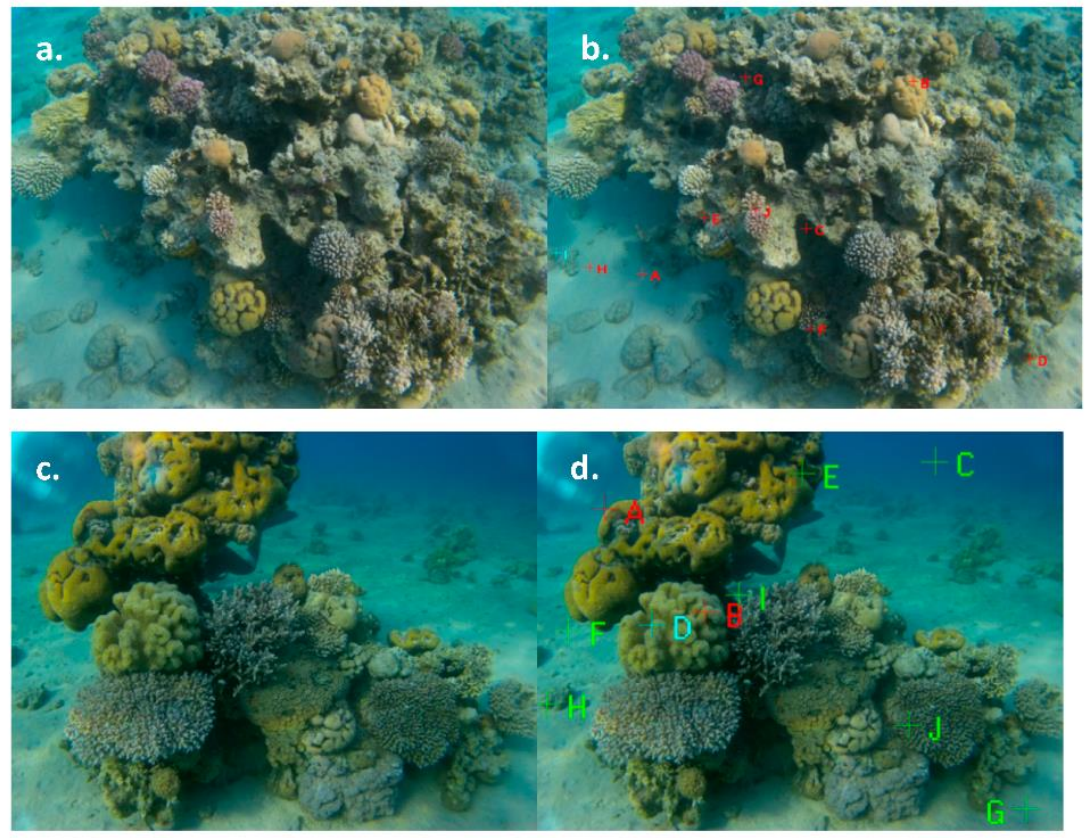

Figure 2. (a) Coral species in the Gulf of Eilat; (b). Coral Point Count software for the annotation process; (c) photograph of an additional spot of the coral reef; and (d) annotation process.

\subsubsection{Sampling Frequency}

Field work was conducted from June 2017 till June 2018.

Transects were photographed every month until June 2018.

The images were analysed using DL on a computer with a Tesla K80 GPU accelerator.

\subsubsection{Cover Percentage}

Live coral cover was checked at the four test sites using the method of line transects. In each photo, the exact counts and cover percentage of the eleven coral species were noted for the number of these coral species per transect and for the percentage of coral coverage of each species. Examination of the photos focused on healthy corals. Count- 
based measurements followed the "center rule" scheme, as suggested by Zvuloni et al. [69].

In this work, only corals with centers lying within the sampling unit are counted, and all other corals are ignored. The advantage of this technique is that the size of a coral does not play any role in the sampling probability, making this method nonbiased in contrast to other, biased, methods and corrections reviewed by Zvuloni et al. [69].

Cover percentage was calculated using Microsoft Excel software and CPCe 4.1 software in order to facilitate the logistics of the manual annotation process. Coral counting was done by Fiji ImageJ software. Statistical analysis was done using R statistics software.

Sample images

Video

\section{Sample video}

Video sequences were filmed from June 2017 till June 2018 at the three sites at different depths ( $5 \mathrm{~m}, 10 \mathrm{~m}, 15 \mathrm{~m}$, and $20 \mathrm{~m}$ ). In order to get separate photos from the videos, QuickTime software was used.

\section{Use of video images}

Underwater videos of the coral reef species from the Gulf of Eilat were filmed in order to produce a large dataset of images. Image blocks of $200 \times 200$ pixel-sized image frames were manually cut to comprise the chosen training dataset of 3850 sub images of some coral species, as shown in Figure 3.

\section{Common coral species}

Image preprocessing

For preprocessing, the images were min-max normalized to be compatible with the network architecture (see Figure 4). After detecting and cropping the coral images, that are scaled to $200 \times 200$ pixels preprocessed and labelled. See Figure 5 for specific images for each coral species. 


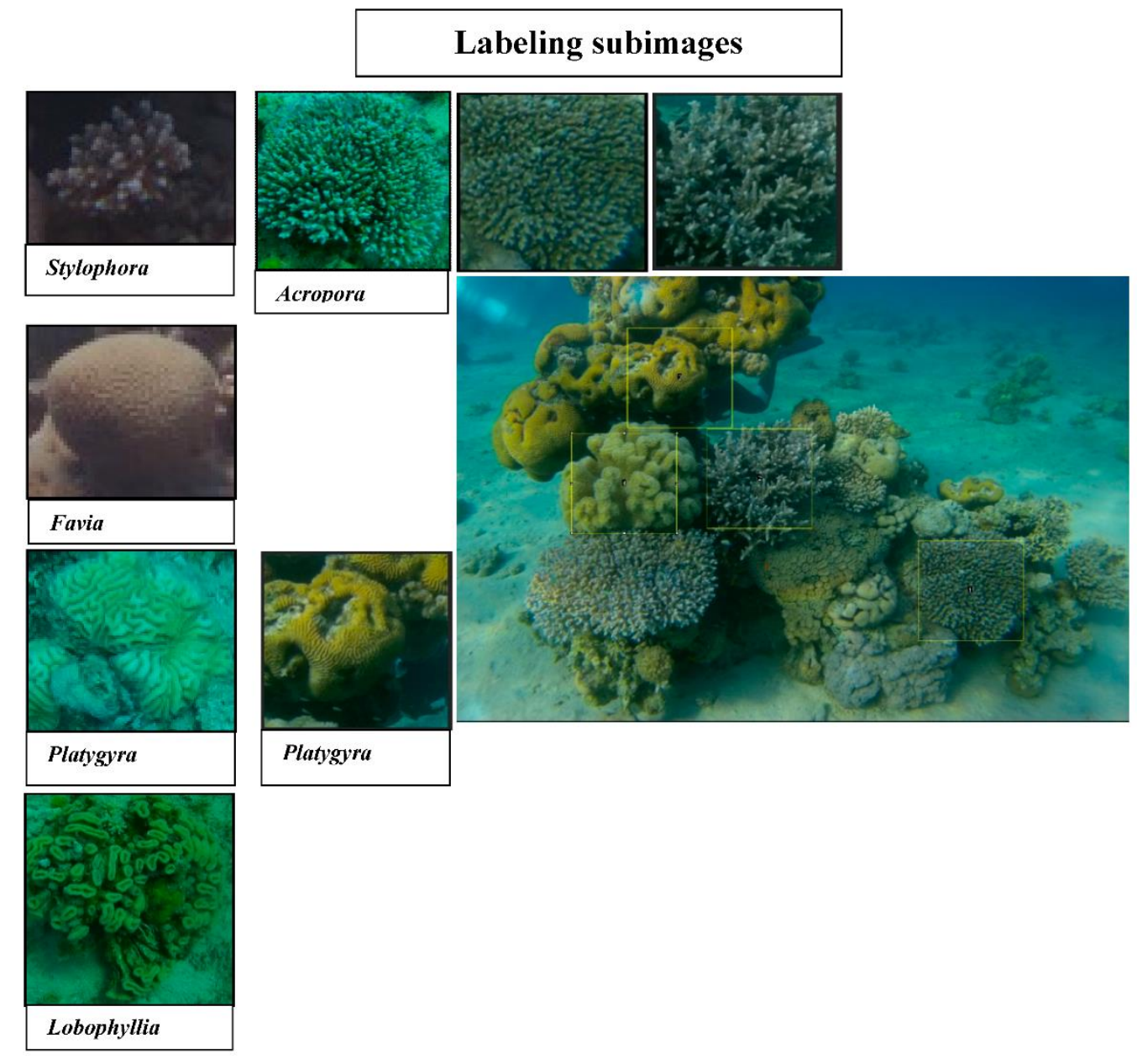

Figure 3. Samples of four of the coral species in the study.

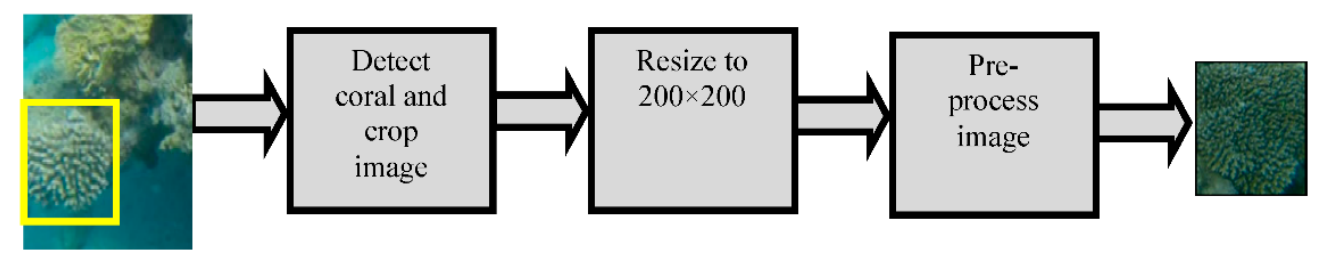

Figure 4. Preprocessing of an image.

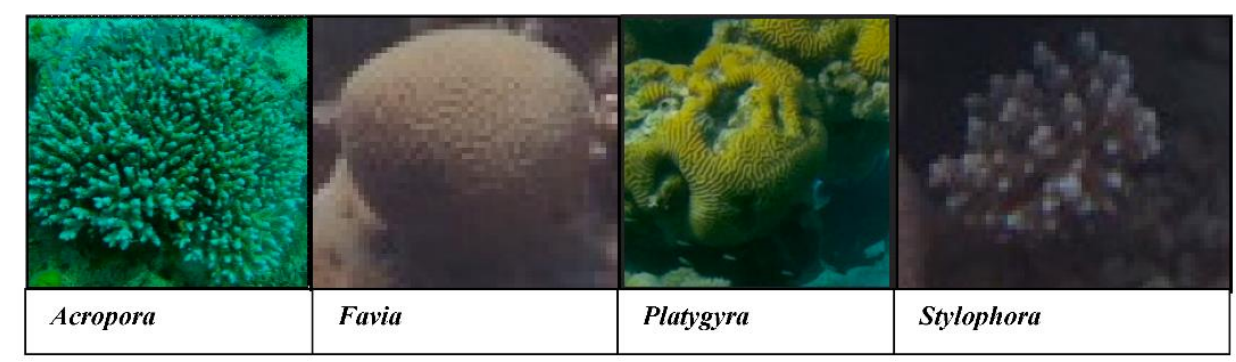

Figure 5. Examples of preprocessed coral images and their labels (from left to right): Acropora, Favia, Platygyra, and Stylophora. 


\section{Results}

\subsection{Obtained by $D L$}

High accuracy of $90 \%$ was obtained in a preliminary test by applying the DL method for classifying 400 images of four common coral species (see Supplementary Table S3).

The method was proven by applying training results from three sites on a fourth external site, reaching accuracy of $80.13 \%$. The fourth site with 2200 images was added classified for eleven coral classes. The results show accuracy of $80.13 \%$ for eleven coral species. The test data results show that the highest accuracy was observed for Stylophora (93.5\%), Lobophyllia (92\%) and Montipora (91.5\%) other species like Platygyra (89.5\%), Acropora (81\%), Cyphastrea (80\%), Porites (74\%), Echinopora (73\%), Pavona (70.5\%), Goniastrea $(69.5 \%)$ were observed in a lower accuracy and the lowest accuracy was found for Favia (67\%) (see Supplementary Table S4).

Using cross-validation results proves that the model can analyse new data from additional sites not used in the training (see Supplementary Tables S6 and S7).

\subsection{Obtained by Traditional, non-DL Methods}

- There is no difference between the methods "Fiji ImageJ" and "Point estimated", applied at each site (Cochran-Mantel-Haenszel test, X2(3) =3.5084, $p=0.3197)$ (see supplementary statistical data Figure S6).

- There is a significant difference among live coral cover and the number of coral colonies for the four sites, by any method (see supplementary statistical data Figure S8).

- The difference in relative species' coverage among the four sites was significant using both methods (see supplementary statistical data Figure S9).

- The species differed significantly in their coverage percentage. The coverage percentage among species differed statistically (One-way ANOVA, $\mathrm{F}(3,12)=11.9, p=$ 0.000657) (see Supplementary Table S5).

\section{Discussion}

The proposed computerized classification method can be configured to different characteristics of the dataset (e.g., size, number of classes, class types, etc.). Several convolutional neural network (CNN) architectures, such as VGG-16 and ResNet-50, were compared by us, and transfer learning was used. We used ResNet-50 on a dataset containing eleven classes of coral species with a large number of images (5500), far more than the amount of images used in other studies.

The classification of underwater coral images is challenging due to the large number of coral species, the great variance among images of the same coral, the lighting conditions, and the fact that several species tend to grow next to each other, leading to increasing overlapping among them. We proved that $\mathrm{CNNs}$ automatic classification of underwater coral images (see Supplementary Table S3) easily outperforms state-of-the-art painstaking manual surveys, of all CNNs, ResNet-50 was the best, due to its relative high speed, and level of accuracy (see Supplementary Table S4).

It is noteworthy that trained technicians or specialists can obviously identify many more than 11 species, as well as delete erroneous data, their training may take years. Furthermore, human workers could never generate the vast amount produced by automated methods. Following the case presented by us, it is obvious that machine based coral survey methods can be expanded to cover any coral species and non-species substrates. We demonstrate the validity of existing automated surveying methods in an environment where such methods were not yet tested.

\section{Transfer Learning}

Due to the challenging problems facing coral reefs exposed to climate change and eutrophication, only DL can provide the vast resources necessary to handle in real time, high resolution enormous amounts of monitoring data. That requires Transfer learning (TL), which 
is an ML procedure that uses a model trained on one task to solve other tasks [70] and additional new problems [71]. That technique works on condition that if the model features learned from the first task are succulently generic to represent the features of the data seen during training.VGG-16 (very deep convolutional networks) is used in pre-trained models due to its high accuracy and advantages over ImageNet based classification.

Lumini et al. [72] used deep learning methods based on $\mathrm{CNN}$ architectures for monitoring underwater ecosystems. In order to do so they used 5 well-known datasets ( 3 of plankton and 2 coral datasets). They showed that their multiple models DenseNet succeeded the performance of the best single models. These authors used experimental data to examine the performance of both the single CNN and the ensemble of CNNs and showed that the best stand-alone model for most datasets is DenseNet.

The DL neural network used here, i.e., VGG-16 (very deep convolutional networks), has been pre-trained and proven on millions of photos. VGG16 has high accuracy and resolves the dataset classification problem inherent in ImageNet.

VGG16 is a 16 layer, $528 \mathrm{MB}$ file size, classification model, based on the ImageNet dataset. It can classify 1000 most different image types such as plants, animals, buildings, and humans.

The improved architecture we used is based on the previously trained VGG-16 network, except for the final three layers.

That architecture resulted in an accuracy of $90 \%$, much better than that obtained by running our images on the original network without any modifications, which would have given very poor results. In addition, the major benefit of using the VGG-16 network was in the remarkably short time required to train the dense layer, which is a fully connected layer in which each unit or neuron is connected to each neuron in the next layer.

\section{Conclusions}

\subsection{The Innovations and Accomplishments of this Study}

- This is the first study of its type done in the Gulf of Eilat.

- Will provide tools to follow the effect of climate change on the coral reefs of the Gulf of Eilat.

- Will allow the establishment of a baseline prior to the opening of the Red-Dead-Canal; real-time monitoring of its effects on the structure and biodiversity of the gulf's coral reefs.

- Foundation benchmark for the benefit of future studies done in the region.

- The refinement and development of the described DL method are applicable to reefs elsewhere.

The accomplishments of our work are by using "big data" in order to address the urgent ecological need of classifying corals, specifically those of the reefs in the Gulf of Eilat. We demonstrated the adaptation and application of Deep Learning Neuronal Networks for classifying corals in the Gulf of Eilat reefs. We applied DL to solve the problem of automated documentation of the structure of the coral reef at four sites in the Gulf of Eilat. Our study includes just corals and yet achieved accuracy similar to those that also included strikingly different classes, such as sea urchins, seaweeds, sand and bare ground.

\subsection{Future Challenges in the Application of DL to the Study of Reefs}

- To develop the capability of DL for the study of time series in order to monitor and reveal temporal changes in the composition of reefs.

- To extract size/age distribution frequencies within single species populations.

- To document changes in live cover of corals in reefs.

Author Contributions: A.R. wrote the manuscript with input from all authors. Z.D. shared his extensive experience and guided the authors with a deeper understanding of the coral reefs and ma- 
rine ecosystems. D.I. actively participated throughout the research process. N.S.N. helped and advised on deep learning throughout the research process. J.I.C.B. helped with presentation of the results and with the preparation for statistical analyses.

Funding: No funding was received for this research.

Data Availability: The datasets generated during and/or analysed during the current study are available from the corresponding author on reasonable request.

Acknowledgments: The authors thank Irit Shoval from the Scientific Equipment Center of the Faculty of Life Sciences at Bar-Ilan University for helping with the data analysis and the subsequent processing of the results. We thank Sharon Victor for her help with formatting the manuscript.

Conflicts of Interest: The authors declare that they have no conflict of interest.

\section{References}

1. Bellwood, D.R.; Hughes, T.P.; Folke, C.; Nystrom, M. Confronting the coral reef crisis. Nature 2004, 429, 827-833.

2. Maor-Landaw, K.; Karako-Lampert, S.; Ben-Asher, H.W.; Goffredo, S.; Falini, G.; Dubinsky, Z.; Levy, O. Gene expression profiles during short-term heat stress in the red sea coral Stylophora pistillata. Glob. Chang. Biol. 2014, 20, $3026-3035$.

3. Teplitski, M.; Krediet, C.J.; Meyer, J.L.; Ritchie, K.B. The Cnidaria Past, Present and Future; Goffredo, S., Dubinsky, Z., Eds.; Springer: Berlin, Germany, 2016; pp. 331-346.

4. Hoegh-Guldberg, O.; Mumby, P.J.; Hooten, A.J.; Steneck, R.S.; Greenfield, P.; Gomez, E.; Harvell, C.D.; Sale, P.F.; Edwards, A.J.; Caldeira, K.; et al. Coral reefs under rapid climate change and ocean acidification. Science 2007, 318, 1737-1742, doi:10.1126/science.1152509.

5. Roberts, J.M.; et al. The Cnidaria, Past, Present and Future; Goffredo, S., Dubinsky, Z., Eds.; Springer: Berlin, Germany, 2016; pp. 593-606.

6. McClanahan, T.R.; Ateweberhan, M.; Muhando, C.A.; Maina, J.; Mohammed, M.S. Effects of climate and seawater temperature variation on coral bleaching and mortality. Ecol. Monogr. 2007, 77, 503-525.

7. Gravili, C. The Cnidaria, Past, Present and Future; Goffredo, S., Dubinsky, Z., Eds.; Springer: Berlin, Germany, 2016 ; pp. 95-107.

8. Dubinsky, Z.; Stambler, N. Marine pollution and coral reefs. Glob. Chang. Biol. 1996, 2, 511-526, doi:10.1111/j.13652486.1996.tb00064.x.

9. Beijbom, O.; Edmunds, P.J.; Kline, D.I.; Mitchell, B.G.; Kriegman, D.J. Automated annotation of coral reef survey images. In Proceedings of the 2012 IEEE Conference on Computer Vision and Pattern Recognition; Institute of Electrical and Electronics Engineers (IEEE), Providence, RI, USA, 16--21 June 2012; pp. 1170-1177.

10. Loya, Y.; Slobodkin, L.B. The coral reefs of Eilat (Gulf of Eilat, Red Sea). Symp. Zool. Soc. Lond. 1971, 28, 117-139.

11. Loya, Y. Community structure and specis diversity of hermatypic corals at Eilat, Red Sea. Mar. Biol. 1972, 13, 100-123.

12. Loya, Y. Possible effects of water pollution on community structure of Red Sea corals. Mar. Biol. 1975, 29, 177-185.

13. Soden, B.J.; Wetherald, R.T.; Stenchikov, G.L.; Robock, A. Global cooling after the eruption of Mount Pinatubo: A test of climate feedback by water vapor. Science 2002, 296, 727-730.

14. Loya, Y.; Lubinevsky, H.; Rosenfeld, M.; Kramarsky-Winter, E. Nutrient enrichment caused by in situ fish farms at Eilat, Red Sea is detrimental to coral reproduction. Mar. Pollut. Bull. 2004, 49, 344-353.

15. Iluz, D.; Yehoshua, Y.; Dubinsky, Z. Quantum yields of phytoplankton photosynthesis in the Gulf of Aqaba (Elat), Northern Red Sea. Isr. J. Plant Sci. 2008, 56, 29-36.

16. Zhang, T.; Zheng, W.; Cui, Z.; Zong, Y.; Yan, J.; Yan, K. A deep neural network-driven feature learning method for multi-view facial expression recognition. IEEE Trans. Multimed. 2016, 18, 2528-2536.

17. Ciresan, D.C.; Meier, U.; Gambardella, L.M.; Schmidhuber, J. Deep, big, simple neural nets for handwritten digit recognition. Neural Comput 2010, 22, 3207-3220.

18. Baldi, P.; Chauvin, Y. Neural networks for fingerprint recognition. Neural Comput. 1993, 5, 402-418, doi:10.1162/neco.1993.5.3.402.

19. Zhang, X.L.; Wu, J. Deep belief networks based voice activity detection. IEEE Trans. Audio, Speech, Lang. Process. 2013, 21, 697710, doi:10.1109/tasl.2012.2229986.

20. Shihavuddin, A.S.M.; Gracias, N.; Garcia, R.; Gleason, A.C.; Gintert, B. Image-based coral reef classification and thematic mapping. Remote. Sens. 2013, 5, 1809-1841, doi:10.3390/rs5041809.

21. Elawady, M. Sparse coral classification using deep convolutional neural networks. Thesis Submitted for the Degree of MSc Erasmus Mundus in Vision and Robotics (VIBOT 2014). arXiv 2015, arXiv:1511.09067v1.

22. Mahmood, A; et al. Oceans 2016; Zande, J., Kirkwood, B., Eds.; IEEE: Piscataway, NJ, USA, 2016; pp. 1-5.

23. Gómez-Ríos, A.; Tabik, S.; Luengo, J.; Shihavuddin, A.; Krawczyk, B.; Herrera, F. Towards highly accurate coral texture images classification using deep convolutional neural networks and data augmentation. arXiv 2018, arXiv:1804.00516.

24. Roberts, C.M.; McClean, C.J.; Veron, J.E.N.; Hawkins, J.P.; Allen, G.R.; McAllister, D.E.; Mittermeier, C.G.; Schueler, F.W.; Spalding, M.; Wells, F.; et al. Marine biodiversity hotspots and conservation priorities for tropical reefs. Science 2002, 295, 1280-1284, doi:10.1126/science.1067728. 
25. Schumacher, H.; Zibrowius, H. What is hermatypic? A redefinition of ecological groups in corals and other organisms. Coral Reefs 1985, 4, 1-9.

26. Brandt, K. Uber die symbiose von algen und tieren. Arch. Anat. Physiol. (Archiv für Anatomie und Physiologie) 1883, 1, $445-454$.

27. Odum, H.T.; Odum, E.P. Trophic structure and productivity of a windward coral reef community on Eniwetok Atoll. Ecol. Monogr. 1955, 25, 291-320.

28. Dubinsky, Z.; Iluz, D. The Cnidaria, Past, Present and Future: The World of Medusa and her Sisters; Goffredo, S., Dubinsky, Z., Eds.; Springer: Berlin, Germany, 2016; pp. 469-487.

29. Goreau, T.F.; Goreau, N.I.; Goreau, T.J. Corals and coral reefs. Sci. Am. 1979, 241, 124-136.

30. Levy, O.; Dubinsky, Z.; Achituv, Y.; Erez, J. Diurnal polyp expansion behavior in stony corals may enhance carbon availability for symbionts photosynthesis. J. Exp. Mar. Biol. Ecol. 2006, 333, 1-11.

31. Goreau, T.F. The physiology of skeleton formation in corals. I. A method for measuring the rate of calcium deposition by corals under different conditions. Biol. Bull. 1959, 116, 59-75.

32. Cuif, J.P. The Cnidaria, Past, Present and Future; Goffredo, S., Dubinsky, Z., Eds.; Springer: Berlin, Germany, 2016; pp. 163-179.

33. Cohen, I.; Dubinsky, Z.; Erez, J. Light enhanced calcification in hermatypic corals: New insights from light spectral responses. Front. Mar. Sci. 2016, 2, doi:10.3389/fmars.2015.00122.

34. Maor-Landaw, K.; Ben-Asher, H.W.; Karako-Lampert, S.; Salmon-Divon, M.; Prada, F.; Caroselli, E.; Goffredo, S.; Falini, G.; Dubinsky, Z.; Levy, O. Mediterranean versus Red sea corals facing climate change, a transcriptome analysis. Sci. Rep. 2017, 7, srep42405, doi:10.1038/srep42405.

35. Anthony, K.R.N.; Kline, D.I.; Diaz-Pulido, G.; Dove, S.; Hoegh-Guldberg, O. Ocean acidification causes bleaching and productivity loss in coral reef builders. Proc. Natl. Acad. Sci. USA 2008, 105, 17442-17446.

36. Fox, H.E.; Pet, J.S.; Dahuri, R.; Caldwell, R.L. Recovery in rubble fields: Long-term impacts of blast fishing. Mar. Pollut. Bull. 2003, 46, 1024-1031, doi:10.1016/s0025-326x(03)00246-7.

37. Wood, E. Collection of Coral Reef Fish for Aquaria: Global Trade, Conservation Issues and Management Strategies; Marine Conservation Society: 2001.

38. Downs, C.A.; Kramarsky-Winter, E.; Segal, R.; Fauth, J.E.; Knutson, S.; Bronstein, O.; Ciner, F.R.; Jeger, R.E.; Lichtenfeld, Y.; Woodley, C.M.; et al. Toxicopathological effects of the sunscreen UV filter, oxybenzone (benzophenone-3), on coral planulae and cultured primary cells and its environmental contamination in Hawaii and the US Virgin Islands. Arch. Environ. Contam. Toxicol. 2016, 70, 265-288, doi:10.1007/s00244-015-0227-7.

39. Longcore, T.; Rich, C. Ecological light pollution. Front. Ecol. Environ. 2004, 2, 191-198.

40. Wielgus, J.; Balmford, A.; Lewis, T.B.; Mora, C.; Gerber, L.R. Coral reef quality and recreation fees in marine protected areas. Conserv. Lett. 2010, 3, 38-44, doi:10.1111/j.1755-263x.2009.00084.x.

41. Littler, M.M.; Littler, D.S.; Brooks, B.L. Harmful algae on tropical coral reefs: Bottom-up eutrophication and top-down herbivory Harmful Algae 2006, 5, 565-585.

42. Hunter, C.L.; Evans, C.W. Coral reefs in Kaneohe Bay, Hawaii: Two centuries of western influence and two decades of data. Bull. Mar. Sci. 1995, 57, 501-515.

43. Hughes, T.P.; Baird, A.H.; Bellwood, D.R.; Card, M.; Connolly, S.R.; Folke, C.; Grosberg, R.; Hoegh-Guldberg, O.; Jackson, J.B.C.; Kleypas, J.; et al. Climate change, human impacts, and the resilience of coral reefs. Science 2003, 301, 929-933, doi:10.1126/science.1085046.

44. Fitt, K.; Brown, B.E.; Warner, M.E.; Dunne, R.P. Coral bleaching: Interpretation of thermal tolerance limits and thermal thresholds in tropical corals. Coral Reefs 2001, 20, 51-65.

45. Gorbunov, M.Y.; Falkowski, P.G. Photoreceptors in the cnidarian hosts allow symbiotic corals to sense blue moonlight. Limnol. Oceanogr. 2002, 47, 309-315.

46. Tamir, R.; Lerner, A.; Haspel, C.; Dubinsky, Z.; Iluz, D. The spectral and spatial distribution of light pollution in the waters of the northern Gulf of Aqaba (Eilat). Sci. Rep. 2017, 7, 42329, doi:10.1038/srep42329.

47. Shlesinger, Y.; Loya, Y. Coral community reproductive patterns: Red Sea versus the Great Barrier Reef Science 1985, 228, 13331335.

48. Zakai, D.; Dubinsky, Z.; Avishai, A.; Caaras, T.; Chadwick, N.E. Lunar periodicity of planula release in the reef-building coral Stylophora pistillata. Mar. Ecol.-Prog. Ser. 2006, 311, 93-102.

49. Beyth, M. The Red Sea and the Mediterranean-Dead Sea canal project. Desalination 2007, 214, 365-371.

50. Berman, T.; Paldor, N.; Brenner, S. Simulation of wind-driven circulation in the Gulf of Elat (Aqaba). J. Mar. Syst. 2000, 26, 349365.

51. Lee, H.; Grosse, R.; Ranganath, R.; Ng, A.Y. In Proceedings of the 26th Annual International Conference on Machine Learning; ACM: 2009; pp. 609-616.

52. Kruszynski, K.J.; Kaandorp, J.A.; van Liere, R. A computational method for quantifying morphological variation in scleractinian corals. Coral Reefs 2007, 26, 831-840.

53. Chindapol, N.; Kaandorp, J.A.; Cronemberger, C.; Mass, T.; Genin, A. Modelling growth and form of the scleractinian coral Pocillopora verrucosa and the influence of hydrodynamics. PLoS Comput. Biol. 2013, 9, e1002849, doi:10.1371/journal.pcbi.1002849.

54. Mahmood, A.; et al. Handbook of Neural Computation; Samui, P., Roy, S.S., Balas, V.E., Eds.; Academic Press: 2017 ; pp. $383-401$. 
55. Mahmood, A.; Bennamoun, M.; An, S.; Sohel, F.; Boussaid, F.; Hovey, R.; Fisher, R.B. September. Automatic annotation of coral reefs using deep learning. In Proceedings of the Oceans 2016 mts/IEEE Monterey, Monterey, CA, USA, 19-23 September 2016; pp. 1-5.

56. Mahmood, A.; Bennamoun, M.; An, S.; Sohel, F.A.; Boussaid, F.; Hovey, R.; \& Fisher, R.B. (2018. Deep image representations for coral image classification. IEEE J. Ocean. Eng. 2018, 44, 121-131, doi:10.1109/joe.2017.2786878.

57. Mahmood, A.; Ospina, A.G.; Bennamoun, M.; An, S.; Sohel, F.; Boussaid, F.; \& Kendrick, G.A. (2020. Automatic Hierarchical Classification of Kelps Using Deep Residual Features. Sensors 2020, 20, 447, doi:10.3390/s20020447.

58. Mahmood, A.; Bennamoun, M.; An, S.; Sohel, F.; Boussaid, F. ResFeats: Residual network based features for underwater image classification. Image Vis. Comput. 2020, 93, 103811.

59. Szegedy, C.; Vanhoucke, V.; Ioffe, S.; Shlens, J.; Wojna, Z. Rethinking the inception architecture for computer vision. In Proceedings of the IEEE Conference on Computer Vision and Pattern Recognition, 2016; pp. 2818-2826.

60. He, K.; Zhang, X.; Ren, S.; Sun, J. Delving deep into rectifiers: Surpassing human-level performance on ImageNet classification. In Proceedings of the IEEE International Conference on Computer Vision, 2015; pp. 1026-1034.

61. Huang, G.; Liu, Z.; Van Der Maaten, L.; Weinberger, K.Q. Densely connected convolutional networks. In Proceedings of the IEEE Conference on Computer Vision and Pattern Recognition, 2017; pp. 4700-4708.

62. Beijbom, O.; Edmunds, P.J.; Roelfsema, C.; Smith, J.; Kline, D.I.; Neal, B.P.; Chan, S. Towards automated annotation of benthic survey images: Variability of human experts and operational modes of automation. PLoS ONE 2015, 10, e0130312, doi:10.1371/journal.pone.0130312.

63. Williams, I.D.; Couch, C.; Beijbom, O.; Oliver, T.; Vargas-Angel, B.; Schumacher, B.; \& Brainard, R. Leveraging automated image analysis tools to transform our capacity to assess status and trends on coral reefs. Front. Mar. Sci. 2019, 6, 222.

64. Loya, Y. Recolonization of Red Sea corals affected by natural catastrophes and man-made perturbations. Ecology 1976, 57, 278289.

65. Loya, Y.; Rinkevich, B. Effects of oil polution on coral reef communities. Mar. Ecol. Prog. Ser. 1980, 3, 167-180.

66. Genin, A.; Lazar, B.; Brenner, S. Vertical mixing and coral death in the Red Sea following the eruption of Mount Pinatubo. Nat. Cell Biol. 1995, 377, 507-510, doi:10.1038/377507a0.

67. Bhattacharya, D.; Agrawal, S.; Aranda, M.; Baumgarten, S.; Belcaid, M.; Drake, J.L.; Erwin, D.; Foret, S.; Gates, R.D.; Gruber, D.F.; et al. Comparative genomics explains the evolutionary success of reef-forming corals. eLife 2016, 5, 5741, doi:10.7554/elife.13288.

68. Shaked, Y.; Genin, A. Annual Report of the Israel National Monitoring Program in the Northern Gulf of Aqaba; Israel Ministry of the Environment: 2018.

69. Zvuloni, A.; Artzy-Randrup, Y.; Stone, L.; van Woesik, R.; Loya, Y. Ecological size-frequency distributions: How to prevent and correct biases in spatial sampling. Limnol. Oceanogr. Methods 2008, 6, 144-153, doi:10.4319/lom.2008.6.144.

70. Yosinski, J.; Clune, J.; Bengio, Y.; Lipson, H. How transferable are features in deep neural networks? Adv. Neural Inf. Process. Syst. 2014, 27, 3320-3328.

71. Pan, S.J.; Yang, Q. A survey on transfer learning. IEEE Trans. Knowl. Data Eng. 2009, 22, 1345-1359.

72. Lumini, A.; Nanni, L.; Maguolo, G. Deep learning for plankton and coral classification. Appl. Comput. Inf. 2020, doi:10.1016/j.aci.2019.11.004. 